\title{
Hazard Minimization and Environmental effect of Chemical Waste produced in our Laboratory-An Educational Article
}

\author{
Kiran Singh, Rajeev K. Shukla and Tripti Singh* \\ Department of Chemistry, VSSD College, Kanpur-208002 (India) \\ Email: singh.tripss@gmail.com
}

\begin{abstract}
The environmental effect caused by the laboratory with regard to chemical waste is of growing issue concern, and attempts to solve the issue are being made constantly. Educational and studies-related establishments, in maximum laboratory and non-laboratory activities, contribute to the generation of small quantities of waste, lots of them are highly toxic. Out of this waste, a few is indexed via government organizations, are worried approximately environmental pollutants: disposal of acids and bases, metals, solvents, chemical substances and toxicity of artificial products, whose toxicity is frequently unknown. This text provides an assessment of the hassle and identifies feasible solutions, indicating pertinent laws, directives and tips. Remedial measures is to be implemented by the institution in order to minimize the generation of waste; waste minimization procedures such as reduction, reuse, recycling, treatment and disposal of chemicals at initial stage. Physical observations and chemical treatments and analysis were carried out in this work by performing many tests and measurements. Fairly good and surprisingly results were obtained.
\end{abstract}

Index Terms: Hazard minimization, Laboratory waste, Environmental effect, Physical and chemical analysis and Treatment methods

\section{INTRODUCTION}

The environmental impact of chemical waste made by chemical laboratory is a topic of great concern and discussion since least two decades[1] on the generation of hazardous wastes. Several of them are highly toxic and require the need of necessity to implement a apply for the treatment of chemical waste in instructional establishments. A number of this is often listed by governmental agencies who are concerned about the quality of the environment. Examples embrace the disposal of toxic acids, metals, solvents, chemicals and also synthesis products of synthesis whose toxicity is usually unknown. what is more, it's noteworthy that the composition of waste from analysis labs perpetually changes consistent with every project being developed. This case will now not be unnoticed by tutorial establishments, and numerous analysis and academic establishment that are involved concerning this down side and are desegregation venturesome waste management into their activities. Several such activities are elaborate out by many workers[2-11]. The work by Nolasco et al[12-13], analyses the implementation of programs for managing laboratory chemical waste in universities, and states that many programs are responding to the wants of the pillars of property and ecological awareness that were the most proposal.

One of the most significant advantages is undoubtedly the fact that students are taught how to adequately deal with the waste produced in research and laboratories, thereby minimizing damage to the environment. Moreover, another advantage, which should not be overlooked is that of working in a safe, healthy and clean environment, in line with the principles of ecology[14-15].As stated above, although the amount of waste generated in academic institutions is small, less than $1 \%$ of the total generated nationally, waste in education institutions is considered heterogeneous, and may include highly toxic compounds. Therefore, any teaching and research institution committed to its employees' and students' health must consistently uphold the laws related to workers' chemical safety, and laws on management of hazardous waste released by its laboratories. The concept of waste minimization encompasses any action that reduces the amount of toxicity to be discarded as hazardous waste. It is therefore essential that the waste is properly handled, stored and disposed of. When the waste generating source has been identified, whether highly hazardous, operational procedures aimed at their appropriate disposal should be implemented. Most of what is used in university laboratories, related to research or teaching at some point, can become hazardous. Examples are solvents, glassware, reagents, packaging of dangerous products, biological material, broken or obsolete equipments, broken thermometers, and obsolete computers. A visitor to most unpretentious academic laboratories would see such material, which can cause safety problems and have an impact on environmental health if disposed of in an indiscriminate manner. Thus, it is important to urgently address this problem, where this can be done by cutting down on waste production, and properly treating and disposing of the waste which is produced. Thus, institutions both private and public can benefit from the implementation of such systems that significantly improve the environment in which 


\section{Available online at www.ijrat.org}

they are located. A program of waste management is an integral part of the environmental care recommended by ISO $14000^{14}$. Such programs can and should be implemented in educational institutions, and this necessarily includes constant evaluation of laboratory activities and processes, aimed at reducing the generation of disposable material and increasing recycling. The hazardous waste should be discarded as such and not as common garbage, seeking to minimize environmental impact and to adhere to specific waste management laws enforced by many legislations[1517].

Hazardous chemicals normally found in academia and requiring proper treatment they are: chemical wastes generated in research laboratories, old chemical agents, often difficult to identify and abandoned in the laboratory, chemical agents surpassing their expiration date and therefore in need of re-evaluation of their effectiveness, and need for disposal, bottles of chemicals without labels or with wrong or unreadable labels, material in a state of deterioration or in packages which are deteriorated, or damaged, unknown residues in chemicals containers, laboratory waste such as paper towels and rags, personal protective equipment, pesticides, equipment containing toxic compounds, different types of waste oils, used solvents, thinner, oil remover, formaldehyde, formalin, acryl amide waste in liquid or gel form, mercury and other metals with high toxicity, thermometers, sharp devices such as: needles, syringes, chromatography needles, bleach, ammonia, cleaning solvents, liquid wood polish, chemical bottles (glass and plastic) empty but contaminated, contaminated broken (or damaged) laboratory glass, mercurycontaminated, broken (or damaged) thermometers, carcinogenic chemicals ect.

Reduction of hazardous chemical waste can be done normally by three processes: Reduce the use of hazardous material and, where possible, use small quantities of chemical agents; Reuse the material, i.e., store it properly to be used when necessary and Recycle which uses filtration and distillation systems, among others, that allow the reuse of solvents. Another important way to minimize waste generation is to consider the use of less toxic chemicals, in chemical laboratories. Avoid the use of unnecessary ingredients, such as emulsifiers in solvents to be used or discarded, and separate the different types of solvents for reuse or recycling. The university laboratories tend to generate a considerable amount of chemical waste as they often use outdated techniques and a large volume of solvents. Other suggestions designed to minimize the generation of waste are: replace the use of mercury thermometers for digital thermometers; replace sulfochromic solution or alcoholic solution of potassium hydroxide, when possible for the cleaning laboratory glassware; replace tests with acids and strong bases, replace carbon tetrachloride by cyclohexane. The aim of this study is to create awareness to the students and laboratory technicians for the reduction of hazardous chemical waste, produced in the laboratory and to assess the physical and chemical analysis of wastewater flowing and their impact on the environment.

\section{EXPERIMENTAL SECTION 2.1 Sampling}

A total of four samples of untreated and contaminated solution were taken from the outlet of the laboratory at different time intervals (approximately 10 days ) when the laboratory was running, for the physical and chemical analysis.

\subsection{Materials}

Excessive purity and AR grade reagents used within the chemical analysis had been received from Merck Co. Inc., Germany and purified via distillation in which the middle fraction turned into amassed. The liquids have been saved in darkish bottles over $0.4 \mathrm{~nm}$ molecular sieves to reduce water content material and had been partially degassed with a vacuum pump. The purity of each compound turned into checked with the aid of fuel chromatography and the outcomes indicated that the mole fraction purity was better than 0.97 . The purity of chemical compounds used become showed by comparing the physical properties with those pronounced inside the literature.

\subsection{Apparatus and Technique}

Before every series of experiments, we calibrated the instruments at atmospheric pressure with doubly distilled water. The electric conductance turned into measured by way of conductivity meter 304-Systronics and the uncertainty observed in the conductance become within $\pm 0.1 \mathrm{dS} / \mathrm{m}$. The $\mathrm{pH}$ turned into measured through virtual $\mathrm{pH}$ meter model MKV1-Systronics and the uncertainty observed in the $\mathrm{pH}$ measurement changed into within \pm 0.01 . The turbidity turned into measured by way of The turbidity turned into measured by way of turbidity meter-Bexco.Shimadzuax-200 correct to within $\pm 0.1 \mathrm{mg}$ become used for all of the weighing functions. Water bath tub, Electrically heated thermostat, Porcelain evaporating dish, Dryer: used silica gel as drying agent, center quantitative filter out paper or filter membrane (diameter of hollow was $0.45 \mathrm{~mm}$ ) had been used. All of the measurements had been executed at $25^{\circ} \mathrm{C}$.

\subsection{Methods}

\subsubsection{Neutralization:}

The most common treatment is to neutralize highly acidic or alkaline solutions, leading to a desirable $\mathrm{pH}$ of 6 to 8.5. Thus, if this solution does not contain other toxic compounds it can be treated as regular trash and discarded in the sewage. Strong acids or bases must be neutralized before being released into the sewage, including those with the following cations: $\mathrm{Sn}^{2+}, \mathrm{Sr}^{2+}$, 


\section{Available online at www.ijrat.org}

$\mathrm{Ti}^{3+}, \mathrm{Ti}^{4+}, \mathrm{Zr}^{2+} ; \quad \mathrm{Al}^{3+}, \mathrm{Ca}^{2+}, \mathrm{Fe}^{2+}, \mathrm{Fe}^{3+}, \mathrm{H}^{+}, \mathrm{K}^{+}, \mathrm{Li}^{+}$, $\mathrm{Mg}^{2+}, \mathrm{Na}^{+},\left(\mathrm{NH}_{4}\right)^{+}, \mathrm{Zn}^{2+}$, and anions: $(\mathrm{OH})^{-}, \mathrm{I}^{-},\left(\mathrm{NO}_{3}\right)^{-}$, $\left(\mathrm{PO}_{4}\right)^{3-},\left(\mathrm{SO}_{4}\right)^{2-},(\mathrm{SCN})^{-},\left(\mathrm{BO}_{3}\right)^{3-},\left(\mathrm{B}_{4} \mathrm{O}_{7}\right)^{2-}, \mathrm{Br}^{-},\left(\mathrm{CO}_{3}\right)^{2-}$, $\left(\mathrm{HSO}_{3}\right)^{-},(\mathrm{OCN})^{-}$,

\subsubsection{Precipitation, oxidation and reduction}

These processes can remove hazardous components of chemical waste and the final product can be discarded as common trash. Precipitates derived from these reactions may require more effective waste treatment. The application of these procedures for chemical treatment in laboratories, apart from reducing hazardous waste, allows its incorporation as a common practice in teaching the students responsible management of chemical waste, fostering future generations of scientists with a better understanding of proper waste disposal.

The recycling of solvents, among other materials used in technical analysis, allows the reuse of material, which otherwise would be discarded as hazardous waste. These techniques require planning, when they are incorporated into the teaching laboratories activities. Solvent recycling, if well done, brings advantages to the academy in terms of risk reduction, harmful waste reduction, lower costs, and is also beneficial for the students because as previously mentioned, it allows them to learn about waste management in a responsible manner and understand the university commitment to hazardous waste reduction.

As mentioned earlier, educational and research institutions generate pollution because they produce harmful waste, discard hazardous materials in the sink, allow the evaporation of solvents, among other activities detrimental to the environment. The recommended process of reducing production of harmful wastes is not always feasible, because the concept of research implies the study of new compounds and their disposal if the result is neither interesting nor relevant. This type of activity is quite different from industrial processes, where there are routine activities, constant use of raw materials and well known waste products. In research, the multitude of non-routine activities is inherently more difficult to control. Nevertheless, the suggestions described here may contribute to the prevention of environmental pollution, and minimize costs with health and the environment.

\subsubsection{Treatment, Storage, And Disposal}

Several options are available for hazardous-waste management. The most desirable is to reduce the quantity of waste at its source or to recycle the materials for some other productive use. Nevertheless, while reduction and recycling are desirable options, they are not regarded as the final remedy to the problem of hazardous-waste disposal. There will • always be a need for treatment and for storage or disposal of some amount of hazardous waste.

\subsubsection{Treatment of Hazardous waste}

Hazardous waste can be treated by chemical, thermal, biological, and physical methods. Chemical ways embrace ion exchange, precipitation, oxidation and reduction, and neutralization. Among thermal ways is high-temperature burning,that not solely will detoxify bound organic wastes however can also destroy them. Special sorts of thermal instrumentation are used for burning waste in either solid, liquid, or sludge form. These embrace the fluidized-bed furnace, multi ple-hearth chamber, rotary oven, and liquidinjection furnace. One drawback display by hazardou s-waste burning is that the potential for pollution. Physical processes embrace evaporation, sedimentation, flotation, and filtration. Yet another process is solidification, which is achieved by encapsulating the waste in concrete, plastic. Encapsulation produces a solid mass of material that is resistant to leaching. Waste can also be mixed with lime, fly ash, and water to form a solid, cement like product.

\section{RESULTS AND DISCUSSION}

\subsection{Characterization of waste}

The great style of laboratory waste makes waste categorization difficult. Transport and waste regulations are written for commercially available high-volume chemicals, which may make it difficult to categorize some laboratory chemicals, such as experimental or newly synthesized materials. Categorization procedures should account for the common laboratory waste management practices of putting little containers of waste chemicals into a bigger over pack drum, and mixing of the many solvents and solutes into one drum of ignitable liquids. There are many acceptable information sources for waste characterization, together with the identity of the supply or raw materials, inlaboratory check procedures (such as those described below), associated analysis by an environmental laboratory. Generator data is used for waste characterization appreciate the data of waste characteristics and constituents by laboratory personnel United Nations agency conducted the method, procedure, or experiment.

\subsection{Definition of Characteristic Waste}

According to federal law, the properties of chemical waste that create hazards are as follows. Note that these definitions are unique, particularly the definition of waste having the characteristic of toxicity.

1.Ignitability: Ignitable materials are outlined as having one or a lot of of the subsequent characteristics: :

liquids that have a flash point of less than $60{ }^{\circ} \mathrm{C}$ or some other characteristic that has the potential to cause fire; 


\section{Available online at www.ijrat.org}

- materials but liquids that are capable, under standard temperature and pressure, of causing fire by friction, adsorption of moisture, or spontaneous chemical changes and, when ignited, burn so vigorously and persistently that they create a hazard;

- flammable compressed gases, including those that form flammable mixtures;

- oxidizers that stimulate combustion of organic materials.

Ignitable materials embrace most common organic solvents, gases such as hydrogen and hydrocarbons, and certain nitrate salts.

2. Corrosivity: Corrosive liquids have a $\mathrm{pH} \leq 2$ or $\mathrm{pH}$ $\geq 12.5$ or corrode certain types of steel. Most common laboratory acids and bases are corrosive. Solid corrosives, such as sodium hydroxide pellets and powders, are not legally considered to be corrosive. However, trained laboratory personnel must recognize that such materials are extremely dangerous to skin and eyes and must be handled accordingly.

3. Reactivity: The reactivity classification includes substances that are unstable, react violently with water, detonate if exposed to some initiating source, or produce toxic gases. Alkali metals, peroxides and compounds that have per-oxidized, and cyanide or sulfide compounds are classed as reactive.

4. Toxicity: Toxicity is established through the toxicity characteristic natural action procedure (TCLP) test, that measures the tendency of bound harmful materials to be leached (extracted) from the waste below circumstances assumed to breed conditions of a lowland a comparatively little variety of industrially necessary harmful chemicals and is predicated on the leachate concentration, higher than that a waste is taken into account venturous. Failure to pass the TCLP leads to classification of a fabric as a waste material. The TCLP check is primarily for solid materials; liquids square measure generally evaluated on a straight concentration basis. TCLP analyses square measure typically performed by environmental testing laboratories.

\subsection{Characterization of Unknowns}

Establishing the hazardous characteristics and evaluating the potential listing of clearly known waste is sometimes quite easy. Unidentified materials gift a drag, however, as a result of use, treatment, and disposal facilities have to be compelled to recognize characteristics and hazards to manage waste safely. All chemicals must be characterized sufficiently for safe transportation offsite. Analysis of laboratory unknowns is expensive, particularly if Environmental Protection Agency ways should be used, or the presence of a constituent should be dominated out, and handling unknowns is risky because of the potential presence of unstable, reactive, or extremely harmful chemicals or byproducts. though high-priced, some waste disposal companies provide on-the-scene services to categorise unknown laboratory waste to organize it for cargo to their treatment facility.

\subsection{In-Laboratory take a look at Procedures for Unknowns}

When the identity of the fabric isn't bestknown, easy in-laboratory take a look at procedures are often administered to work out the hazard category into that the fabric ought to be classified. as a result of the generator could also be ready to provide some general data, it's going to be helpful to hold out the take a look at procedures before the materials ar off from the laboratory. Perform these tests given that they'll be done safely, and given that they facilitate the characterization of the waste needed by your risky waste disposal firm. Perceive that the subsequent take a look at procedure are solely to supply further data, and don't meet independent agency restrictive needs for waste analysis.

In general, exactly determinative the molecular structure of the unknown material isn't necessary. Hazard classification sometimes satisfies the restric tive needs and people of the treatment disposal facility. However, it's necessary to determine that analytical information are needed by the disposal facility. Trained laboratory personnel United Nations agency perform the analytical procedures ought to be at home with the characteristics of the waste and any necessary precautions. as a result of the hazards of the materials being tested are un known, the tilization of correct personal protection and safety devices like chemical hoods and shields is imperative. Older samples are notably dangerous as a result of they'll have modified in composition, to illustrate, through the formation of peroxides. the subsequent data is often needed by treatment and disposal facilities before they conform to handle unknown materials: The following take a look at procedures ar pronto accomplished by trained laboratory personnel. the general sequence for testing for liquid and solid materials is as; Physical description. embrace the state of the fabric (solid, liquid), the color, and also the consistency (for solids) or viscosness (for liquids). For liquid materials, describe the clarity of the solution (transparent, clear, or opaque). If associate degree of unknown material may be a bi- or tri-layered liquid, describe every layer singly, giving associate degree approximate proportion of the whole for very layer, when taking acceptable

safety precautions for handling the unknown, together with the utilization of non-public protection devices, take away a tiny low sample to be used within the following tests.

Water reactivity: Add a small quantity of the unknown to a few millilitres of water. Observe any changes, including heat evolution, gas evolution, and fame generation. 


\section{Available online at www.ijrat.org}

Water solubility. Observe the solubility of the unknown in water. If it's associate degree insoluble liquid, note whether or not it's less or a lot of dense than water (i.e., will it float or sink?). Most non halogenated organic liquids are less dense than water.

pH: Take a look at the fabric with multirange hydrogen ion concentration paper. If the sample is soluble, take a look at the hydrogen ion concentration of a tenth solution. finishing up a neutralization volumetric analysis may be fascinating or maybe needed.

Ignitability (flammability); Place a tiny low sample of the fabric $(<5 \mathrm{~mL})$ in associate degree metallic element take a look at receptacle. Apply associate degree ignition supply, generally a gas torch, to the take a look at sample for 0.5 second.If the fabric supports its own combustion, it's a ignitable liquid with a flash purpose of under $60^{\circ} \mathrm{C}$. If the sample doesn't ignite, apply the ignition supply on for once for second. If the fabric burns, it's flammable. Flammable materials have flash purpose between 60 and $93^{\circ} \mathrm{C}$.

Presence of oxidizer: Wet commercially on the market starch-iodide paper with one $\mathrm{N}$ acid, and place a tiny low portion of the unknown on the wetted paper. A amendment in color of the paper to dark purple may be a positive take a look at for associate degree of oxidizing agent. The take a look at can even be administered by adding 0.1 to 0.2 $\mathrm{g}$ of of sodium or potassium iodide to $1 \mathrm{~mL}$ of an acidic $10 \%$ solution of the unknown. Development of a amber color indicates associate degree of oxidizing agent. To check for hydro peroxides in waterinsoluble organic solvents, dip the starch-iodine take a look at paper into the solvent, and let it dry. Add a drop of water to constant section of the paper. Development of a yellow-brown color indicates an oxidizer. To test for hydroperoxides in water-insoluble organic solvents, dip the starch-iodine test paper into the solvent, and let it dry. Add a drop of water to the same section of the paper. Development of a dark color indicates the presence of hydro-peroxides.

Presence of peroxides: The following tests detect most of the peroxy compounds, including all hydro peroxides.Peroxide test strips, which turn to an indicative color in the presence of peroxides, are available commercially. Note that these strips should be air dried till the solvent evaporates and exposed to wet for correct operation. Add 1 to 3 $\mathrm{mL}$ of the liquid to be tested to an equal volume of acetic acid, add a few drops of 5\% aqueous potassium iodide solution, and shake. The appearance of a yellow to brown color indicates the presence of peroxides. Alternatively, addition of $1 \mathrm{~mL}$ of a freshly prepared $10 \%$ solution of potassium iodide to $10 \mathrm{~mL}$ of an organic liquid in a $25-\mathrm{mL}$ glass cylinder produces a yellow color if peroxides are present.
Add $0.5 \mathrm{~mL}$ of the liquid to be tested to a mixture of 1 $\mathrm{mL}$ of $10 \%$ aqueous potassium iodide solution and 0.5 $\mathrm{mL}$ of dilute hydrochloric acid to which has been added a few drops of starch solution just prior to the test. The appearance of a blue or blue-black color within 1 minute indicates the presence of peroxides.None of these tests should be applied to materials (such as metallic potassium) that may be contaminated with inorganic peroxides.

Presence of sulfide: Commercial test strips for the presence of sulfide are available, and their use is recommended. If the test strips are not available in the laboratory, the subsequent take a look at are often performed. Warning: This takea look at produces risky and odiferous vapors. Use solely little quantities of resolution for the take a look at and use acceptable ventilation. The take a look at for inorganic sulfides is administered only if the hydrogen ion concentration of associate degree solution of the unknown is bigger than ten. Add a couple of drops of focused acid to a sample of the unknown whereas holding a chunk of economic sugar of lead paper, wet with water, over the sample. Development of a brown-black color on the paper indicates generation of sulfide.

Presence of cyanide: business check strips for the presence of cyanide area unit on the market, and their use is powerfully suggested.

Presence of halogen: Heat a bit of copper wire till red in a very flame. Cool the wire in distilled or deionized water, and dip it into the unknown. once more heat the wire within the flame. The presence of group is indicated by a inexperienced color round the wire within the flame.

The aim of this study is to assess the physical and chemical properties of laboratory wastewater stored in tank and after treatment these properties were compared with the treated waste water. Four samples of laboratory waste water were collected at different time intervals approximately 10 days each, and a rough analysis of physical and chemical properties were done and listed in Table. The results of study on some physical and chemical properties of untreated and treated waste water showed that: The $\mathrm{PH}$ values were ranges from 11.9 - 10.3 for untreated wastewater, 9.3 - 8.8 for treated. Turbidity was $67.5-65.3$ JTU for untreated wastewater, 51.2 - 50.7 JTU for treated wastewater. The electrical conductivity for untreated sample ranges from 8.2 - $7.8 \mathrm{mS} / \mathrm{cm}, 5.9$ - $5.3 \mathrm{mS} / \mathrm{cm}$ for treated sample. Results indicated that there were significant differences in total dissolved solids among the treated and untreated sample. It was found that the differences in BOD and COD between treated wastewater and untreated wastewater were significant . The highest values of BOD and COD while the lowest values of BOD and COD were recorded. Insignificant observations were recorded except untreated wastewater for the presence of ions like sulfide, halogens, peroxides and cyanide. The major 


\section{Available online at www.ijrat.org}

mineral elements content of treated water were $\mathrm{Ca}$, $\mathrm{Mg}, \mathrm{Na}, \mathrm{K}$ and $\mathrm{P}$. The trace element present in treated water was $\mathrm{Cr}, \mathrm{Cu}, \mathrm{Fe}, \mathrm{Zn}, \mathrm{Pb}, \mathrm{Ni}$ and $\mathrm{Mn}$,

\subsection{Recommendations:}

In the process of waste reduction at supply, the goal is to facilitate any activity that reduces the generation of venturous chemical waste. This activity can be enforced with sensible management once getting materials; once replacement harmful material with less harmful ones, and with sensible laboratory apply. Here are some suggestions that enable the reduction of waste at source:

-Implement a policy of minimizing waste within the students apply laboratory, and train all those concerned in the activities. There reduction at the supply will be achieved through improvement of strategies and replacement of ineffective

instrumentation. In instructional establishments, this can be not continually attainable, however one ought to think about using modern extraction techniques, comparable to solid section extraction, or critical fluid, to attenuate waste by victimizations smaller volumes of organic solvents.

- Do not mix dangerous categories of waste with nonhazardous ones.

- Consider the possibility of using less toxic reagents, substituting for products with lower toxicity. In this context, it is During this context, it's attainable to think the replacement of products such as benzene, used as a solvent, with hexane or xylene; formalin or formaldehyde, used as a preservative for specimens within the laboratory, with ethanol; halogenated solvents in the extraction process with non-halogenated solvents; sodium dichromate with sodium hypochlorite, in some oxidation reactions; replace liquid scintillation-based toluene with a nonflammable solvent; in qualitative tests for heavy metals, replace sulfide ion with ion hydroxide. Replacement isn't continually attainable as a result of some substitutes always produce fully satisfactory results, or are toxic or too expensive. Thus, it is necessary to check if the replacement material is suitable and delivers acceptable results. A common practice is to make an inventory of the compounds used in laboratories and to identify the likely replacement. The laboratory technician answerable for the employment of those compounds has to measure the attainable replacements, victimization the data given by suppliers within the material safety data sheet.

- Centralize the acquisition of chemicals, biological and radiation materials.

- Dating all received material, thus facilitating earlier use of the oldest ones.

- Create a listing of purchased and used chemical agents within the laboratory: maintain a file containing their location, that ought to be updated annually. This facilitates the reduction of the number keep, and acquisition of unnecessary material.

-Offer workers with updated MSDS material Safety knowledge Sheet of the chemicals employed in laboratories.

-Acquire any chemical,biological and radiation materials within the least attainable quantity. It's safe and environmentally correct to shop for less material, use less and, ultimately, get rid of less, permitting a discount in risk of accidents, fires, or hurt to human health, and at constant time reduces prices.

- Purchase the equipment needed for immediate use and avoid getting materials in large quantities, even it looks to be economically advantageous, since stocking can be expensive, or dangerous, and may lead to products exceeding their expiration date. A major part of disposal done by universities is expounded to the acquisition of unnecessary instrumentation.

-Label all reagents to permit their prepared identification. Borrow material from different labs, or pass in little quantities.

-Take into account of testing in micro-scale, using new glassware and techniques that reduce quantities used to milligrams, which yields many benefits such as lower costs, since small-scale experiments using fewer solvents and other chemical agents are generally processed more quickly, because it is faster to heat or cool small volumes, reduces exposure to harmful agents and reduces harmful emissions. However, please note that this technique can only be implemented to achieve the analytical proposed objective.

-Take into account the choice of demonstrations on video, computer modeling and simulations, that eliminate environmental impacts, as substitutes for laboratory tests within the class room. These multimedia simulations allow the student to observe more complex procedures than would be possible in traditional activities in the laboratory.

- Avoid victimization reagents containing toxic metals such as lead, chromium, arsenic, mercury, barium, silver, cadmium and selenium.

- Do not use sulfo chromic solutions: substitute them for less toxic solutions such as biodegradable detergents. Evaluate the possibility of using hot water and detergent for cleaning glass, instead of solvents;

- Always keep the laboratory clean and in order.

-Discard waste for disposal within the sink resulting in the system. Some organic and inorganic compounds is discarded within the sewage works. Generally, soluble organic compounds that have lower boiling temperature of roughly $50^{\circ} \mathrm{C}$ should $n^{\prime} t$ be discarded during this manner. The compounds listed below are readily biodegradable and may be discarded within the sink: Organic compounds: alkyl radical alcohols with not upto five carbon atoms:tamyl alcohol; Alkanediols with not upto eight carbon atoms: glycerin, sugars alcoxy alkanes with not upto seven carbon atoms, 2-Chloroethanol; Aldehydes: open-chain compounds with not upto 5 


\section{Available online at www.ijrat.org}

carbon atoms; Amides with not upto 5 carbon atoms,Amines: open-chain compounds with not upto 7carbonatoms; open-chain diamines with not upto 7 carbon atoms, pyridine carboxylic acids, category of ammonia salts, $\mathrm{Na}$ and metal salts of the acids, Esters: esters with not upto 5carbon atoms; isopropyl acetate. The compounds that have unpleasant odor, comparable to dimethylamine, 1,4-butanedia mine, saturated fatty acid and pentanoic acids should be neutralised and their salts ought to be discarded within the sink into waste drain diluted with at least 1,000 volumes of water; Ketones with not upto 6carbonatoms; Nitriles: acetonitrile, Propionitrile; Sulfonicacid: Na or metal salts of those acids area unit acceptable.

\section{CONCLUSION}

Several countries are encouraging the implementation of this concept in industries and activities of research, including rewarding companies and researchers for developing chemical processes, services and products that not damage the environment. However, the relevance of green chemistry is to incorporate their concerns into the concept of danger or toxicity of chemicals. It is value noting that, the concept of toxicity is the potential capability that the substances have to present a hazard to life and the environment under certain conditions. Thus, in avoiding this hazard you can make use of the basic paradigm of Toxicology that is the concept of reduction, recycling and reuse of the waste. This approach allows the intrinsic toxicity of chemical compounds and also their conditions of exposure to be dealt with. Physical and chemical properties of waste water such as; color, odor, flammability, water solubility, corrosivity, reactivity, electrical conductivity, $\mathrm{pH}$ and turbidity have been checked and toxicity has been estimated by TDS, COD and BOD measurements and several test has also been performed for the detection of toxic ions such as; sulfide, peroxides, cyanide, halogens and the presence of oxidizer. From the results listed in table, it is concluded that after rigorous treatment of the waste water, the treated water is still unsafe for the human life as well as for the ecological environment. Further, it is suggested that the implementation of constant training of university teachers and students with regard to safety in the use, storage and disposal of dangerous products, is key for all above mentioned procedures of health and management quality to become a reality.

\section{Acknowledgement}

Authors are thankful to all faculty members of chemistry department and technical staff of VSSD College for cooperation and for providing information's and facilities.

\section{REFERENCES}

[1] P.C. Ashbrook and P.A. Reinhardt. "Hazardous Wastes in Academia". Environ. Sci. Technol., 19: 1150-1155, 1985.

[2] C.J.O. Cunha. "Laboratory Waste Management Program of the Chemistry Department of UFPR". Quim. Nova, 24: 424-427, 2001.

[3] J.C. Afonso, L.A. Noronha, R.P. Felipe and N. Freidinger. "Laboratory Waste Management: Recovery of Elements and Preparation for final disposal". Quim. Nova, 26: 602-611, 2003.

[4] S.T. Amaral, P.F.L. Machad, M.C.R. Peralba, R. Camara and T.Santos. "Report of an Experiment: Recovery and Registration of Waste from the Undergraduate Laboratories of the Institute of Chemistry of the Federal University of Rio Grande do". Sul. Quim. Nova, 24: 419- 423, 2001.

[5] L.B. AlberguinI, L.S. Silva and M.A.O. Rezende. " Laboratory Chemical Waste USP São Carlos - Results of the Pioneering Experience in Waste Management and Management in a University field ". Quim. Nova, 26: 291-295, 2003.

[6] J.A. Bendassolli, F. Máximo and G.A. Tavares, Ignoto, R. F. "Management of Chemical Residues and Wastewater in the Laboratory of Stable Isotopes of CENA / USP ". Quim. Nova, 26: 612-617, 2003.

[7] A.E. Gerbase, F.S. Coelho, P.F.L. Machado and V.F. Ferreira. "Chemical Waste Management in Educational and Research Institutions ". Quim. Nova, 28: 3, 2005.

[8] I. Imbroisi, A.J.M. Guaritá-Santos, S.S. Barbosa, S.F. Shintaku, H.J. Monteiro, G.A. East Ponce, J.G. Furtado, C.J. Tinoco and D.C. Mello. "Management of Chemical Residues in Universities: Brasília University in focus ". Quim. Nova, 29: 404-409, 2006.

[9] W.F. Jardim. "Management of chemical residues in teaching and research laboratories ". Quim. Nova, 21: 671-673, 1998.

[10]M.A. Armour. "Hazardous Laboratory Chemicals Disposal Guide ". Boca Raton: Lewis, 546, 1996 .

[11] Z.T.C. Leite, S. Alcantara and J.C. Afonso. "The Management of Laboratory Waste in the view of students of a Chemistry Undergraduate course and Related areas". Quim. Nova, 31: 1892-1897, 2008.

[12] F.R. Nolasco, G.A. Tavares and J.A. Bendassolli. "Implementation of Laboratory Chemical Waste Management Programs at Universities: Critical Analysis and Recommendations ". Eng. Sanit. Ambient., 11:118-124, 2006.

[13] M.H Otenio, A.O. Santos, M.F.M. Guimarães, C.C.M. Otenio and C.P. Nogueira. "Management of Biological Waste in Scientific Research Institutions. A case study". Infarma, 20: 35-40, 2008.

[14] D.R. Rondinelli, G. Vastag and G. Panacea. "Common sense, or just a label?: The value of ISO 14001 Environmental Management Systems ". Eur. Manag. J., 18: 499-510, 2000. 


\section{Available online at www.ijrat.org}

[15] J. Dhote, A. Chavhan and S. Ingole. "Design of Laboratory Based Waste Water Treatment Plant. Int. Res. J. of Science \& Engineering, 2 (3): 104-111, 2014.

[16] M. Archer, T. Balf, S. Howard, Z.S. Graham and R. Stuart. "The new England Laboratories Project XL:
An experiment in laboratory regulation ". Chem. Heal Saf., 7: 32-40, 2000 .

[17] D.J. Monz and F.M. Mcdonough, "EPA's proposed Academic Laboratories Rule: A more Flexible Approach to the Management of Hazardous Waste ". J. Chem. Health. Saf., 13: 5-8, 2006. 
International Journal of Research in Advent Technology, Vol.7, No.3, March 2019

E-ISSN: 2321-9637

Available online at www.ijrat.org

Table Physical and Chemical analysis of untreated and treated samples of laboratory waste water at $25^{\circ} \mathrm{C}$.

\begin{tabular}{|c|c|c|c|c|c|c|c|c|}
\hline Physical observations & \multicolumn{4}{|c|}{ Untreated } & \multicolumn{4}{|c|}{ Treated } \\
\hline & Sample 1 & Sample 2 & Sample 3 & Sample 4 & Sample 1 & Sample 2 & Sample 3 & Sampl4 \\
\hline Colour & $\begin{array}{l}\text { Dark } \\
\text { brown } \\
\text { yellowish }\end{array}$ & $\begin{array}{l}\text { Dark } \\
\text { brown } \\
\text { yellowish }\end{array}$ & $\begin{array}{l}\text { Dark } \\
\text { brown } \\
\text { yellowish }\end{array}$ & $\begin{array}{l}\text { Dark brown } \\
\text { yellowish }\end{array}$ & $\begin{array}{l}\text { Brown with } \\
\text { yellowish } \\
\text { green }\end{array}$ & $\begin{array}{l}\text { Brown with } \\
\text { yellowish } \\
\text { green }\end{array}$ & $\begin{array}{l}\text { Brown with } \\
\text { yellowish } \\
\text { green }\end{array}$ & $\begin{array}{l}\text { Brown with } \\
\text { yellowish } \\
\text { green }\end{array}$ \\
\hline Odor & Foul smell & Foul smell & Foul smell & Foul smell & Foul smell & Foul smell & Foul smell & Foul smell \\
\hline Turbidity(JTU) & 67.5 & 65.3 & 65.9 & 66.2 & 51.2 & 50.9 & 51.0 & 50.4 \\
\hline Alkalinity & 24 & 28 & 27 & 26 & 22 & 23 & 21 & 22 \\
\hline Flammability & Low & Low & Low & Low & Inflammable & Inflammable & Inflammable & Inflammable \\
\hline Corrosivity & High & High & High & High & Low & Low & Low & Low \\
\hline Reactivity & High & High & High & High & Low & Low & Low & Low \\
\hline $\mathrm{pH}$ & 10.3 & 11.6 & 10.9 & 10.6 & 8.8 & 9.3 & 9.1 & 9.1 \\
\hline $\begin{array}{l}\text { Electrical conductivity } \\
(\mathrm{mS} / \mathrm{cm})\end{array}$ & 7.3 & 8.2 & 7.8 & 7.4 & 5.3 & 5.9 & 5.6 & 5.5 \\
\hline TDS (mg/l) & 2700 & 2900 & 2650 & 2700 & 2100 & 2200 & 2050 & 2000 \\
\hline COD mg/l & 2440 & 2350 & 2320 & 2410 & 2020 & 2170 & 2080 & 2110 \\
\hline BOD mg/l & 300 & 310 & 330 & 310 & 200 & 190 & 200 & 210 \\
\hline
\end{tabular}

